\title{
Validation of the Fixation of Amorphous Magnetic Glass-coated Magnetic for Biomedical Applications
}

\author{
Irenej Poláček ${ }^{1 *}$, Radovan Hudák ${ }^{1}$, Marek Schnitzer' ${ }^{1}$ Jozef Živčák ${ }^{1}$ \\ ' Department of Biomedical Engineering and Measurement, Faculty of Mechanical Engineering, Technical University of Kosice, Letna 9, 04200 \\ Kosice, Slovakia
}

\begin{abstract}
SSufficient control of the mechanical stress and temperature at the tissue-implant interface after the process of implantation is an important part of validation of the implantation and acceptance of the implant by the internal environment of the body. For this purpose being able to measure these values without contact and with the smallest possible interference in the implant is important. For these measurements the use of amorphous magnetic glass-coated microwires (AMGCM) is very promising.

In comparisons with the sensors which are currently used in intelligent implantations, e.g. RFID sensors, which have dimensions on the order of tens of $\mathrm{cm}$ and are relatively expensive, AMGCM have a great advantage in sizes (less than 50 $\mu \mathrm{m}$ in diameter and for a functional sensor a length of $5 \mathrm{~mm}$ is minimally necessary) and their manufacture is very inexpensive.

This work is focused on the control of fixation of an AMGCM in a sample of the manufacturing technology Direct Metal Laser Sintering. In addition to the metal magnetic core itself, the fixation of an AMGCM has, due to the magneto-elastic effect, an important influence on the magnetic characteristics of the microwire used. The volume of the fixation material and thus also the working force on the microwire influences the sensitivity of the microwire and influences its Curie temperature (TC), which is an important variable in preparation of the composition of the metal core of the microwire.
\end{abstract}

Keywords: amorphous magnetic glass-coated microwires, metrotomography, magnetoelasticity, magnetostriction, switching field, implant, fixation, microsensors, smart implants.

\section{Introduction}

Intelligent implants, which would be capable of measuring different physical parameters, typically temperature and mechanical stress, have been under development since the late 1980s. The biomechanical centre CHARITÉ $[4,5]$ has since 1989 been developing intelligent implants working with RFID sensors likewise as the newer Microstrain systems [2] using low-frequency RFID sensors [3]. Recently, it has also been working on research and development of a new type of sensor which uses an optical fibre with FBG (Fiber Bragg Grating) sensors. Thus, the use of amorphous magnetic glass-coated microwires (AGCM) is a completely new method of sensing biomechanical parameters from the surroundings of an implant.

So that it is possible to measure temperature and mechanical stress in 
biomedical applications using amorphous glasscoated microwires several factors influencing the magnetic behaviour of the microwire need to be considered, such as, for example:

- chemical composition of the metal core of the microwire,

- method of fixation of the microwire,

- mechanical stress affecting the microwire,

- thermal processing,

- length of the microwire,

- modification of the ends of the microwire.

Since basic research is involved, in our work we thus far only devote ourselves to primary questions, namely to the chemical composition of the metal core of the microwire, which insures us the necessary characteristics of the microwire (positive magnetostriction, magnetic bistability, amorphous structure and a Curie temperature around $50^{\circ} \mathrm{C}$ ), and the method of fixation of the microwire, which is the main subject of this work, because during fixation mechanical forces affect influences the magnetoelastic effect and thus also the sensitivity to mechanical stress and temperature.

\section{Materials and methods}

\subsection{Microwires with positive magnetostriction}

As was already mentioned in the introduction, microwires with positive magnetostriction can be distinguished by bistable magnetic behavior, which is a great advantage. Therefore, the magnetization behaviour of these microwires consists in a single rapid Barkhausen jump which occurs with achievement of a critical field HSW; this field is possible to measure by a simple induction method (Fig.1) [1] and the already mentioned influences work on it.

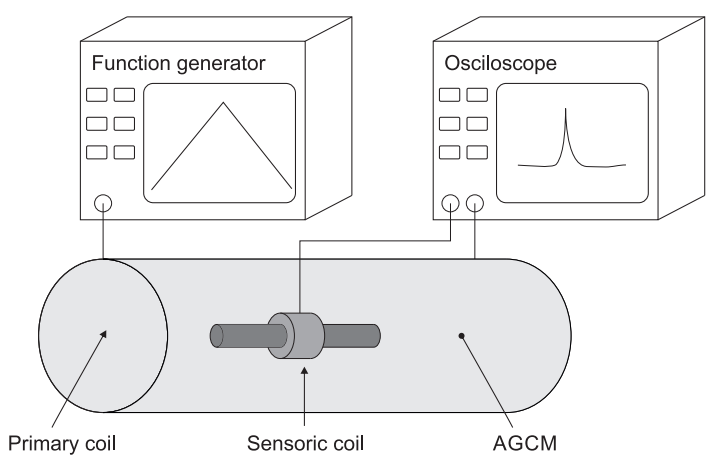

Fig. 1: Apparatus for sensing of a critical field [1].
With generation of a critical field by a generator, the domain wall runs through the microwire. This rapid "run", that is the Barkhausen jump, indicates stress on the sensing coil, which is expressed as a sharp local extreme on the oscilloscope.

\subsection{Influence of temperature on critical fields}

The size of the critical field is determined by the potential of the domain wall. Several factors influence the form of the potential of this wall, such as defects in the core, anisotropism, applied mechanical stress and the like. From this it follows that the thermal dependence of these factors will determine the thermal dependence of the potential of the domain wall; thus, the thermal dependence of the critical field is determined by the thermal dependence of the potential of the domain wall [1].

With rising temperature additional stress is induced thanks to the different coefficient of thermal tensibility of the core and of the glass coating on the microwire, which has as a consequence a magnetoelastic effect. Because of the missing magnetic crystal anisotropism the magnetoelastic effect causes a greater contribution to the critical field, which is determined by the relation [1]:

$\mathrm{HSW} \approx\left(\frac{\lambda_{\mathrm{s}} \sigma}{\mathrm{M}_{\mathrm{S}}^{2}}\right) \cdot \frac{1}{2}$

where: Hsw: critical field $[\mathrm{A} / \mathrm{m}], \lambda_{\mathrm{s}}$ : magnetostriction, $\sigma$ : mechanical stress $[\mathrm{Pa}], \mathrm{Ms}$ : saturation magnetization $[\mathrm{A} / \mathrm{m}]$,

from which it is evident that the critical field is indirectly proportional to the saturation magnetization $\mathrm{Ms}_{\mathrm{s}}$ and directly proportional to magnetostriction $\lambda_{s}$ and the working mechanical stress $\sigma$, which with the changing of the temperature is given mainly by the different temperature tensibility of the glass coating and the metal core. Further, with rising temperature it is easier to achieve the critical field, or the critical field is lower, because with increased temperatures the atoms and their magnetic moments oscillate more, and therefore it is easier to magnetize them [1].

With magnetic materials, microwires as well as the Curie temperature (TC) are also important. The Curie temperature is the temperature above which a material loses its magnetic characteristics; 
however, this process is reversible upon again cooling of the magnet. Just below the Curie temperature, or the Curie point, magnetic characteristics, and thus all processes linked with this, are very sensitive to a change in temperature; that is, even the value of the critical field, as can be seen in Fig. 2. Moreover, a decline in the critical field should be linear for the given segment.

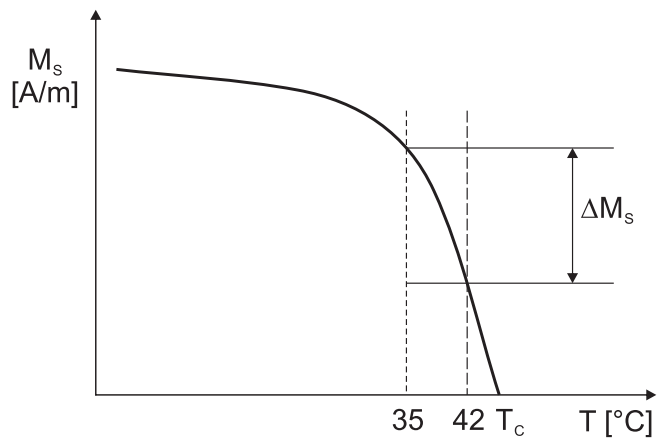

Fig. 2: A depiction of the usable area for measuring temperature just below the Curie temperature (author).

For biomedical purposes (use in implantology) it is necessary that the microwire be sensitive to a temperature from $35-42^{\circ} \mathrm{C}$ with the greatest precision, which means that the Curie temperature should range around $50^{\circ} \mathrm{C}$. The Curie temperature is extremely dependent on chemical composition. It is possible to modify this thermal limit and sensitivity of the microwire by thermally processing the microwire, its length, by applying external forces or modification of the ends of the microwire, for easier tearing away of the domain wall.

\subsection{Manufacture of the microwire}

The amorphous glass-coated microwires with a metal core which were used in our experiments were made using the Taylor-Ulitovski method [6]. The T-U method is the only method for the production of glass-coated microwires with a diameter in $\mu \mathrm{m}$.

The compositional structure of the microwire is obtained by a single step in the process of production. A homogenous foundry alloy is placed into a test tube, which is closed and filled with argon. This foundry alloy in the shape of a bead or its segments must have the correct size, most 3-4 $\mathrm{mm}$ in diameter, so that the bead is embedded at the site of the greatest power of a high-frequency coil.

\subsection{Method of measuring}

Measurements were carried out at the Institute of Physics, Faculty of Sciences, at P.J. Šafárik University in Košice, in the magnetism laboratory.

Since the required composition of the FeMo6, 7, 8B6Cu1 microwire did not have the necessary bistable characteristics and the methods for their creation are slow, for time reasons, a Fe78W5B17 microwire was used which has a Curie temperature of around $170^{\circ} \mathrm{C}$. However, for our purposes of preliminary measurements, with the goal of determining the most suitable method of fixation, it is suitable.

Through measurements, the influence of the method of attaching the Fe78W5B17 microwire to a Ti64 disc, simulating the implants, was examined; this is produced by the method of direct metal laser sintering in the company CEIT-KE (on an EOSINT M280 instrument) at the critical field HSW and at the same time its thermal dependence. Measurements were performed at temperatures from $30^{\circ} \mathrm{C}-170^{\circ} \mathrm{C}$ with a step of $10^{\circ} \mathrm{C}$.

The microwire was attached to the sample in four ways (Fig. 4):

a) At one end,

b) At two ends,

c) In the middle,

d) Along the entire length.
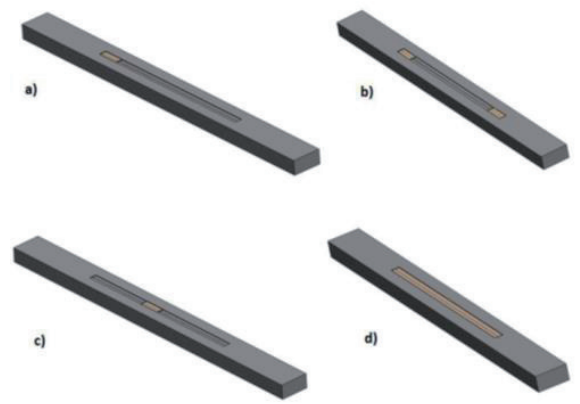

Fig. 3: Method of fixation: a) at 1 end, b) at 2 ends, c) in the middle, d) along the entire length.

\subsection{Control of fixation of the microwire}

From the results of the performed measurements it follows, with a comparison of the thermal dependence of the fixed and unfixed microwire that the optimal method of fixation, from the mentioned four, is along the entire length of the microwire. However, because the magnetic characteristics of the microwire the volume of 
the deposited fixation material influences other sensitivity of the microwire. Upon application a significantly larger volume of the fixation material it was not possible to measure the critical fields, which means that the forces developed by the fixation material during hardening broke the domain structure and the microwire lost its necessary magnetic bistability.

From the mentioned it follows that it is necessary to determine the exact volume of the fixation material and thus also the forces working on the microwire.

For determination of the volume an industrial computer tomograph on a ZEISS Metrotom 1500 device (Germany) was used. The goal was to select the titanium plate, the fixation material and the microwire according to the density. Then to determine the volume of the applied fixation material and to control the course of the microwire in the groove of a plate on a 3D model.

The entry parameters for the measuring of 14 plates and for one plate:

Table 1:Measurements input parameters.

\begin{tabular}{|l|l|l|}
\cline { 2 - 3 } \multicolumn{1}{c|}{} & 14 pieces & 1 piece \\
\hline Voltage $[\mathrm{kV}]$ & 190 & 190 \\
\hline Current $[\mu \mathrm{AA}]$ & 250 & 90 \\
\hline $\begin{array}{l}\text { Integration Time } \\
{[\mathrm{ms}]}\end{array}$ & 2000 & 2000 \\
\hline Gain & 16 & 16 \\
\hline Distance $[\mathrm{mm}]$ & 250 & 60 \\
\hline Voxel $[\mu \mathrm{m}]$ & 67 & 16,5 \\
\hline
\end{tabular}

\section{Results}

The first measurements were performed at one time with fourteen plates with a fixated microwire. With respect to the density of the used material (a titanium alloy Ti64), there was as a consequence of the measuring many artifacts, and the obtained detail did not allow the monitoring of the course of the microwire in the groove.

A limiting factor is also the scanning distance, which with a larger number of scanned plates was greater than with a single piece. With increasing distance of the scanned object to the source of radiation (an $x$-ray tube) the size of the voxel (the volume of a pixel) grows and the enlargement drops upon impact of the radiation on the detector and thus also the amount of details which can be obtained.

With our chosen combination of current and voltage for the 14 plates we achieved a voxel value of $67 \mu \mathrm{m}$.

Since the diameter of the microwire is $50 \mu \mathrm{m}$ and the volume of the fixation material, or its thickness, also moves in the hundreds of $\mu \mathrm{m}$, the value of the achieved voxel does not provide sufficient detail for our requirements, and thus for checking the course of the microwire and determination of the volume of the fixation material.
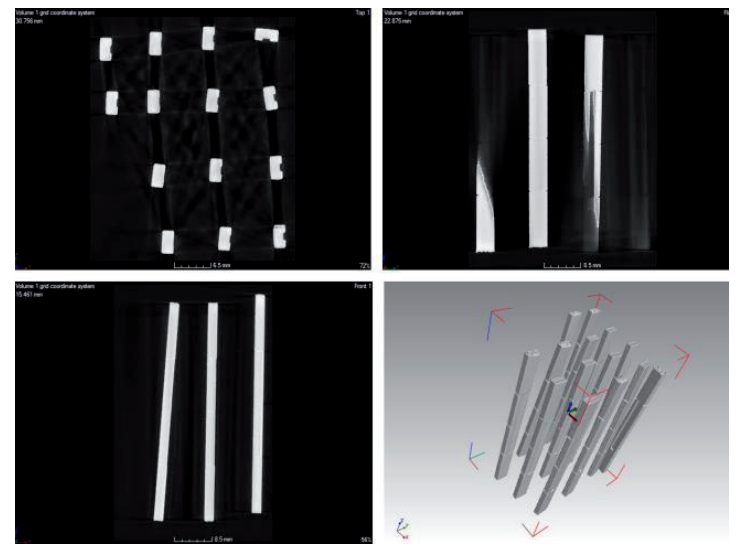

Fig. 4: Measuring of fourteen plates.

For achieving the necessary detail 1 plate was scanned a second time. With respect to the scanning of the segment from the entire plate it was possible to lower the scanning distance and achieve a voxel larger by $16.5 \mu \mathrm{m}$.

These settings and the achieved voxel were on the margin of maximum detail for metal materials that the Metrotome 1500 is capable of achieving. Even this relatively high detail does not allow determination of the exact volume of the fixation material, Fig. 6.

However, with a size of the voxel of $17 \mu \mathrm{m}$ it is possible to use the Metrotom for verification of the presence of the microwire and validation of the process of fixation of the microwire. The line of the microwire can be followed as on a 3D model, Fig. 7, and in the grooves of the individual levels, Fig. 8.

\section{Conclusions}

The use of amorphous magnetic glass-coated microwires as sensors for the scanning of selected physical parameters at the interfaces of tissue- 

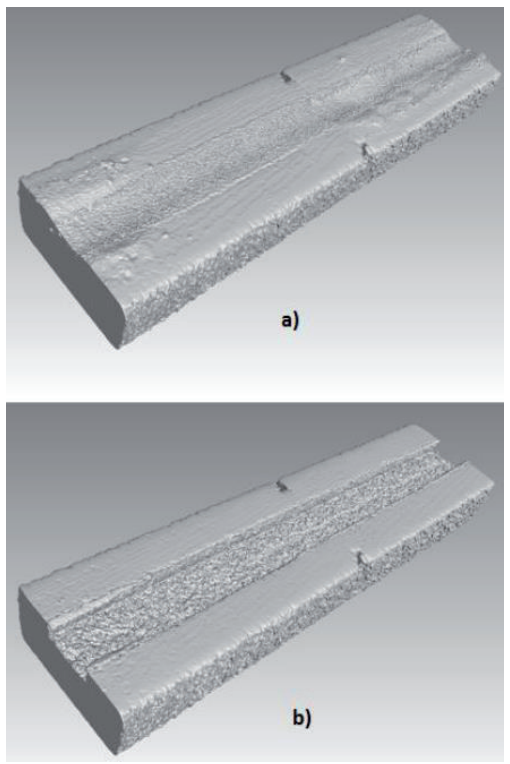

Fig. 5: a) a scan of a plate with the fixation material b) the plate with the software-separated fixation material.

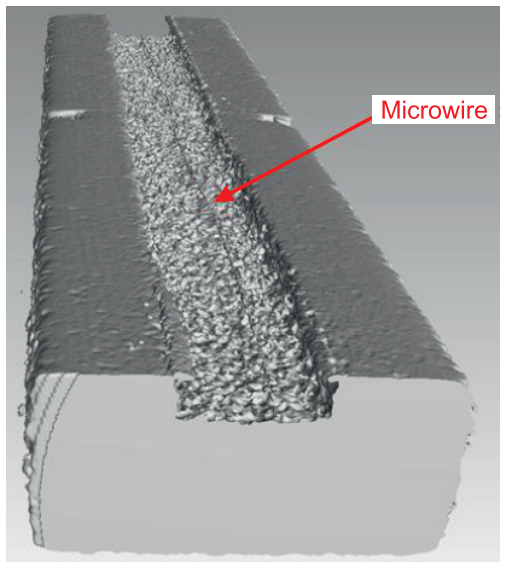

Fig. 6: 3D scan of a plate with a visible microwire.

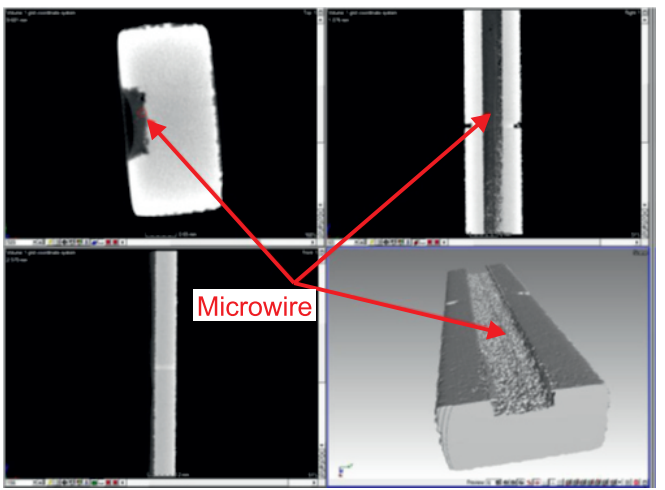

Fig. 7: Scans of plates in 3D view and in the individual levels with a visible microwire. implants is very promising, but thus far the development of these sensore is only on the level of basic research.

Externally applied forces developed by hardening and shrinkage of the fixation material play a significant role in the sensitivity of the sensor. Therefore it is important to determine and control exactly the volume of the fixation material used.

For this purpose the industrial CT ZEISS Metrotom 1500 was selected; however, the technical parameters and limits of the instrument do not permit determining exactly the volume of the fixation material used. With maximum achieved differentiation, however, it was possible to monitor the course of the microwire in the groove of the plate and thus validate the process of microwire fixation.

For more detailed scanning of a plate cooperation with the Institute of Measurement Sciences of the Slovak Academy of Sciences is planned, where their instrumentation will allow scans with exactness up to $0.5 \mu \mathrm{m}$.

\section{Acknowledgements}

This work was supported by the Slovak Research and Development Agency under the contract No. APVV-14-0294.

\section{References and Notes}

[1] Varga, R., Magnetization processes in glass/coated microwires with positive magnetostriction, Institute of Physics, Slovak Academy of Sciences, Bratislava (2012), 413 - 508, DOl: 10.2478/v10155-012-0002- 5,

[2] SW. Arms, CP. Townsend, Wireless Strain Measure-ment Systems - Applications and Solutions Proceed-ings of NSF - ESF Joint Conference on Structural Health Monitoring, Strasbourg, France, October 3-5, 2003,

[3] Ch. Moss, W. Sass, N. Weinrich, K. Seide, J. Müller, (2009) Low Frequency RFID - Strain Measurement on Passive Implants. Proc SOMSED'09 1: 97-100,[4],

[4] P. Damm, V. Schwachmeyer, J. Dymke, A. Bender, G. Bergmann, In vivo hip joint loads during three methods of walking with forearm crutches. Clin Biomech (Bristol, Avon). 2013; DOI: 10.1016/j.clinbiomech.2012.12.003,

[5] G. Bergmann, F. Graichen, A, Rohlmann, A. Bender, B. Heinlein, G. Duda, MO. Heller, MM, Morlock, Realistic loads for testing hip implants. Biomed Mater Eng. 2010; DOI: 10.3233/ BME-2010-06016

[6] A. V. Ulitovski, Method of continunous fabrication of microwires coated by glass (authors certifications (USSR patent), No 128427, 1950) 


\section{Biographical notes}

Irenej Poláček, Ing., received M.S. degree in biomedical engineering from Technical University of Košice, Slovakia in 2013. Today Mr. Polácek is a PhD Student in Biomdeical Engineering at Department of the Biomedical Engineering and Measurement at the Faculty of Mechanical engineering at the Technical University of Košice, Slovakia. His research interests include biomedical engineering, biomechanics, smart implants and amorphous magnetic glasscoated microwires. He is co-author of 5 journal and conference papers.

Radovan Hudák, doc. Ing., PhD., (was born in 1976 in Košice, Slovakia. He received his MS and PhD degrees in biomedical engineering at the Technical University in Košice in 2000 and 2008. Today Mr. Hudák is an associate professor and head of the prosthetics and orthotics study program and head of the Biomedical Engineering Division at the Department of Biomedical Engineering and Measurement, Technical University of Košice. His research interests include additive manufacturing in medicine, medical thermography, industrial thermography and human biomechanics. Since 2004 he has been the technical assistant at the Centre of Refractive Surgery in Košice. Mr. Hudák has more than 100 publications in domestic and foreign journals. He is co-author of 5 monographs and 10 books.

Jozef Živčák, Dr.h.c. prof. Ing., PhD., is a professor of biomedical engineering at the Technical University in Košice. He was born in 1958. He received his MS and PhD degrees from the Technical University of Košice in 1995. Since 2009 he has been a Doctor Honoris Causa of Uzhorod National University, Ukraine. His research interests include human biomechanics, medical sensors and rehabilitation technology. Today he is head of the Department of Biomedical Engineering and Measurement. Since 1998 he has been an expert witness in machine and electrical technology. He is co-author of more than 280 publications in domestic and foreign journals as well as 9 monographs and 12 books. 